temperature was lower in $20 / 30$ patients, max decrease $1,3^{\circ} \mathrm{C}$ (before $37.1^{\circ} \mathrm{C}\left(36.3-37.9^{\circ} \mathrm{C}\right)$ vs. $36.6^{\circ} \mathrm{C}\left(35.9-37.4^{\circ} \mathrm{C}\right)$ ).

Conclusions Early MRI scanning using an MRI incubator is a relatively safe procedure in clinically stable infants. Use of sedation was not associated with clinically relevant changes, although these findings warrant further investigation.

\section{0-163 DOES EARLY SCREENING LEAD TO HIGHER PREVALENCE OF PAEDIATRIC DELIRIUM?}

E Ista, $\mathrm{H}$ te Beest, M de Hoog, D Tibboel, M van Dijk. Intensive Care Unit, Erasmus University Medical Center - Sophia Children's Hospital, Rotterdam, Netherlands

\subsection{6/archdischild-2014-307384.230}

Introduction Early screening of paediatric delirium (PD) allows for early intervention if necessary. The aim of this study was to determine if early screening with the SOS-PD scale led to higher prevalence of PD in ICU patients.

Methods A prospective before-after study design was applied in a population of children aged $>3$ months and admitted for $\geq 48$ $\mathrm{h}$ to the PICU. In the before-period the prevalence of PD was estimated in terms of the number of children with PD confirmed by the consulting psychiatrist. During the after-period nurses systematically assessed the children with the SOS-PD scale three times a day in addition to the psychiatric consultation (SOS-PD score $\geq 4$ ).

Results 148 and 150 children were included in the before and after period, respectively. The prevalence of PD was $6.1 \%$ and $8.7 \%$ for the before and after period respectively (see Table). The relative risk of PD with early screening was 1:43 (95\% CI 0.63 to 3.23 ). In 33 patients (22\%) the SOS-PD score was $\geq 4$ on one or more occasions. In 14 of these patients, the child psychiatrist was consulted. In the remaining patients the child psychiatrist was not consulted for the following reasons: only once a high score $(n=9)$, adverse effects of sedatives $(n=4)$, and underlying disease/motor restlessness $(n=6)$.

Abstract 0-163 Table 1 Patient characteristics and prevalence of PD

\begin{tabular}{l|c|c|c}
\hline & $\begin{array}{c}\text { Before period - usual } \\
\text { care }(\mathrm{N}=148)\end{array}$ & $\begin{array}{c}\text { After period - early } \\
\text { screening PD }(\mathrm{N}=150)\end{array}$ & p-value \\
\hline Gender (F/M) & $59 / 89$ & $76 / 74$ & 0.06 \\
\hline Age (months) & $37(15-124)$ & $54(14-146)$ & 0.22 \\
\hline Length of stay ICU (days) & $7(4-13)$ & $6(4-11)$ & 0.11 \\
\hline Prevalence of PD & $9(6.1 \%)$ & $13(8.7 \%)$ & 0.40 \\
\hline $\begin{array}{l}\text { Number of assessments per } \\
\text { child* }\end{array}$ & - & $9(4-19)$ & - \\
\hline
\end{tabular}

Conclusions Systematic early screening of PD resulted in a higher incidence of PD and could contribute to timely start of treatment.

\section{Allergology}

\section{0-164 SPECIFIC IMMUNOGLOBULIN E TO ARA H 2 AS PREDICTOR FOR PEANUT ALLERGY IN CHILDREN IN A GENERAL DUTCH HOSPITAL}

${ }^{1} \mathrm{M}$ Schots, ${ }^{2} \mathrm{AC}$ de Mol, ${ }^{2} \mathrm{YM}$ Roosen, ${ }^{3} \mathrm{HJ}$ Vermeer, ${ }^{2} \mathrm{AW}$ Vriesman. ${ }^{1}$ Paediatrics, VU Medical Center, Amsterdam, Netherlands; ${ }^{2}$ Paediatrics, Albert Schweitzer Hospital, Dordrecht, Netherlands; ${ }^{3}$ Clinical Chemistry, Albert Schweitzer Hospital, Dordrecht, Netherlands

10.1136/archdischild-2014-307384.231
Background Specific immunoglobulin E (sIgE) to Ara h 2 is described as a potential factor for diagnosing peanut allergy in children. However for the Dutch children, limited data are available. In this study the diagnostic value of sIgE to Ara h2 for children in a general non-university hospital is evaluated and compared with the existing data.

Methods Data from 137 peanut sensitised children were collected retrospectively. The primary outcome was peanut allergy or tolerance confirmed by food challenges. Different possible predictors, including sIgE to Ara h $2(\mathrm{n}=52)$, were identified by multivariate backward stepwise logistic regression analysis. All significant predictors were combined in a formula for prediction of peanut allergy. Different essential cut-off points were obtained by an ROC curve.

Results Multivariate analysis resulted in $\operatorname{sgE}$ to Ara h 2 as only predictor for peanut allergy, with a discriminative ability of 0.87 (95\% CI, 0.77-0.97). Sensitivity and specificity values of respectively 55\% and 95\% were found at a sIgE to Ara h 2 cut-off value of $4.25 \mathrm{kU} / \mathrm{L}$. Hundred percent specificity was reached at a cut-off point of $5.61 \mathrm{kU} / \mathrm{L}$. The mean (SD) sIgE to Ara h 2 level for allergic children was $21.49 \mathrm{kU} / \mathrm{L}$ (SD 30.65) compared to $1.07 \mathrm{kU} / \mathrm{L}(1.56)$ for tolerant children ( $\mathrm{p}=0.001)$.

Conclusions Specific IgE to Ara h 2 is the best predictor for peanut allergy in sensitised children in a non-university hospital, comparable to previously published data. These results are a step forward to a generalisation to the Dutch children population.

\section{0-165 PRENATAL EXPOSURE TO ENDOCRINE DISRUPTING CHEMICALS (EDCS) IS RELATED TO ALLERGIC SYMPTOMS IN 12 MONTH OLD CHILDREN}

${ }^{1}$ I Quaak, ${ }^{1} \mathrm{LC}$ Roosendaal, ${ }^{1} \mathrm{M}$ De Cock, ${ }^{2} \mathrm{MH}$ Lamoree, ${ }^{1} \mathrm{M}$ Van de Bor. ${ }^{1}$ Health and Life Sciences, VU University, Amsterdam, Netherlands; ${ }^{2}$ Institute for Environmental Studies, VU University, Amsterdam, Netherlands

\subsection{6/archdischild-2014-307384.232}

Background In daily life, we are exposed to thousands of manmade chemicals. Some of these chemicals do have endocrine disrupting properties. Prenatal exposure to EDCs may interrupt the maturation of the immune system and lead to childhood allergies.

Objective To determine the relationship between prenatal exposure to EDCs and allergic symptoms in 12 month old children.

Methods Fourty-two pregnant women were recruited at the first antenatal visit to the midwife. Exposure to four different classes of EDCs was determined in cord blood and breast milk: perfluorinated alkyl acids, PCBs, organochlorine pesticides and phthalate metabolites. Allergic symptoms at the age of 12 months was assessed by means of questionnaires. Gender, maternal BMI, parental education and parity were taken into account as possible confounders. Logistic regression analyses were carried out.

Results A significant positive relation was found between prenatal PFOS exposure and allergic symptoms in children at the age of 12 months (OR 4.84; $\mathrm{p}=0.04$ ). In addition, prenatal exposure to PFOA and MECPP was positively related to allergic symptoms in 12 month old children, while a negative association was found for phthalate metabolites MEHHP and MEOHP. However, these associations were not significant (Table 1).

Discussion Prenatal exposure to PFOS is significantly related to allergic symptoms at the age of 12 months. Moreover, associations between exposure to several other EDCs and allergic symptoms in children of 12 months old have been found. In 


\section{Abstract 0-165 Table 1}

\begin{tabular}{llll}
\hline & Odds-ratio & Confidence interval (95\%) & p-value \\
\hline PFOS & 4.84 & $1.11-21.17$ & 0.04 \\
PFOA & 1.85 & $0.39-8.66$ & 0.44 \\
PCB-153 & 1.04 & $0.05-20.42$ & 0.62 \\
P,P'-DDE & 1.00 & $0.99-1.01$ & 0.66 \\
MECPP & 2.01 & $0.26-15.61$ & 0.50 \\
MEHHP & 0.20 & $0.02-1.73$ & 0.15 \\
MEOHP & 0.18 & $0.01-2.34$ & 0.19 \\
MEHP & 0.98 & $0.93-1.03$ & 0.38 \\
\hline
\end{tabular}

conclusion, prenatal exposure to endocrine disrupting chemicals poses children at risk of developing allergic symptoms.

Funding Co-funded by the European Commission within the Seventh Framework Programme DENAMIC (no. 282957).

\section{0-166 WITHDRAWN}

\section{0-167 BENEFICIAL EFFECTS OF SHORT-CHAIN GALACTO - AND LONG-CHAIN FRUCTO-OLIGOSACCHARIDES, BIFIDOBACTERIUM BREVE AND GLUTAMINE ON FOOD ALLERGY-INDUCED BEHAVIOURAL CHANGES IN MICE}

${ }^{1} \mathrm{CGM}$ de Theije, ${ }^{1} \mathrm{Y}$ Borre, ${ }^{2} \mathrm{~N}$ Bartke, ${ }^{2} \mathrm{IB}$ Renes, ${ }^{2,3} \mathrm{RM}$ van Elburg, ${ }^{1,2 \mathrm{~J}}$ Garssen, ${ }^{1} \mathrm{~A}$ Kraneveld. ${ }^{1}$ Pharmacology, Utrecht University, Utrecht, Netherlands; ${ }^{2}$ Nutricia Research, Nutricia, Utrecht, Netherlands; ${ }^{3}$ Pediatrics, VU University Medical Center, Amsterdam, Netherlands

\subsection{6/archdischild-2014-307384.233}

Background and aims Recent studies reveal an important link between the intestinal immune system, microbiota, brain and behaviour. Previously we have shown that food allergy in male mice caused behavioural and neurochemical changes. This study

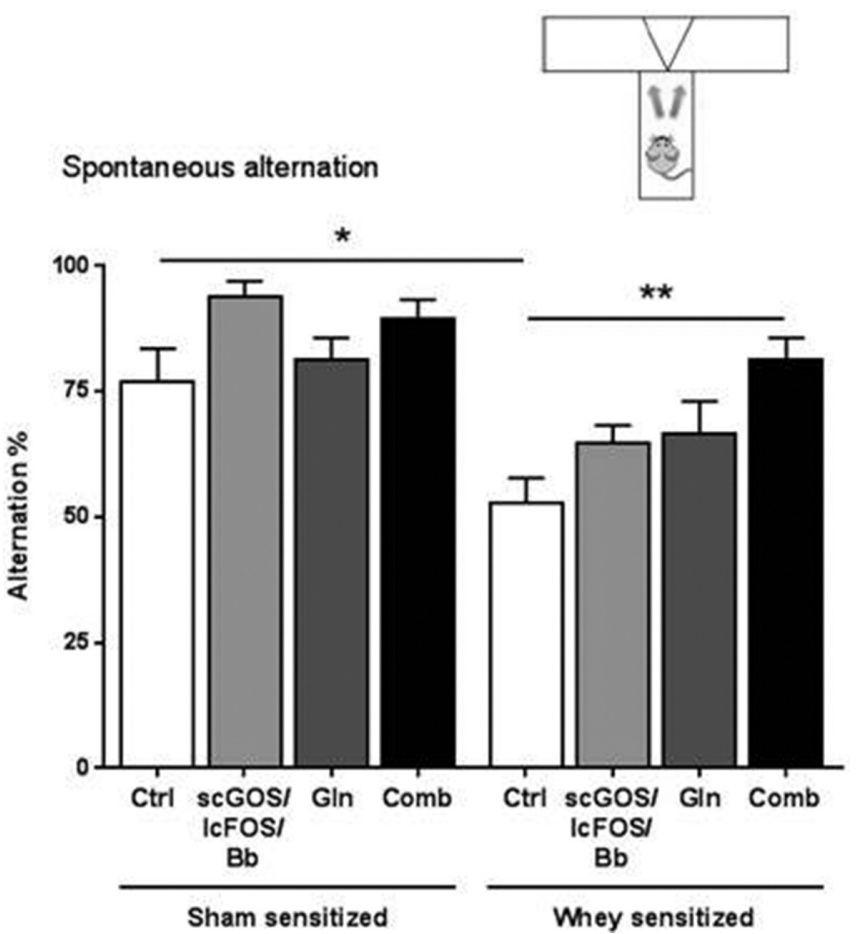

Abstract 0-167 Figure 1 aimed to investigate the effects of a dietary intervention with immunomodulatory short-chain galacto - and long-chain fructooligosaccharides (scGOS/lcFOS), Bifidobacterium breve $(\mathrm{Bb})$ and glutamine (Gln) on behavioural impairments in food allergic mice. Methods Male C3H mice were fed a control, scGOS/lcFOS/Bb, $\mathrm{Gln}$, or scGOS/lcFOS/Bb/Gln (comb) diet shortly after weaning and 2 weeks prior to first sensitisation with whey and cholera toxin (CT), or CT alone. Mice were sensitised for 5 weeks and subsequently orally challenged. Spontaneous alternation was examined in a $\mathrm{T}$ maze test 2 days after the last sensitisation and a social interaction test was conducted 1 day after oral challenge. Spontaneous alternation was used to measure exploratory behaviour and spatial memory.

Results Supplementation with scGOS/lcFOS/Bb or Gln partially prevented reduced spontaneous alternation, whereas supplementation with scGOS/lcFOS/Bb/Gln completely normalised alternation. Both scGOS/lcFOS/Bb and Gln partially attenuated reduced social behaviour in food allergic mice. No additional effect of the combination was observed on social behaviour. Supplementation with scGOS/lcFOS/Bb and/or Gln did not reduce allergic sensitisation, measured by whey-specific immunoglobulins.

Conclusions Supplementation with scGOS/lcFOS/Bb or Gln partially prevented food allergy-induced behavioural impairments and the combination normalised impaired alternation, without changing allergic sensitisation. Therefore, it is of interest to further investigate the effects of dietary supplementation with scGOS/lcFOS/Bb and Gln on immune-induced behavioural impairments in infants.

\section{0-167a INFLAMMATORY SUBTYPES IN WHEEZING INFANTS: ASSESSMENT AND IDENTIFICATION USING INDUCED SPUTUM}

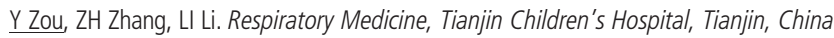

\subsection{6/archdischild-2014-307384.234}

Background Patterns of wheezing during early childhood may indicate differences in aetiology and prognosis of respiratory illnesses.

Objectives This study evaluated sputum cytology in infants with recurrent wheezing to classify sputum inflammatory phenotypes and assessed their characterisation over time.

Methods Sputum induction were performed in 890 infants with recurrent wheezing. Samples were classified as eosinophilic ( $>2.5 \%$ eosinophils), neutrophilic ( $>54 \%$ neutrophils), mixed granulocytic $(>2.5 \%$ eosinophils, $>54 \%$ neutrophils), or paucigranulocytic ( $\leq 2.5 \%$ eosinophils, $\leq 54 \%$ neutrophils). Sputum induction were repeated after 3 months in infants with oral montelukast sodium (4 mg, QN) or nebulizer ICS (Budesonide aerosol $0.5 \mathrm{mg}$, Bid).

Results Total 504 infants (58.1\%)had raised levels of inflammatory cells, eosinophilic $30.6 \%$, neutrophilic $65.2 \%$, mixed granulocytic $4.2 \%$. Variabilities in sputum inflammatory phenotype were observed in both the severe and the mild to moderate wheezing groups. Changes in phenotype were not related to inhaled ICS or oral montelukast sodium, nor were it reflected in a change in tidal pulmonary function. About $27.3 \%$ infants fulfilled the criteria for eosinophilia and there were no differences in severity even atopy between non-eosinophilic and eosinophilic wheezing.

Conclusions Raised levels of inflammatory cells were frequently found in infants with recurrent wheezing. Sputum inflammatory 